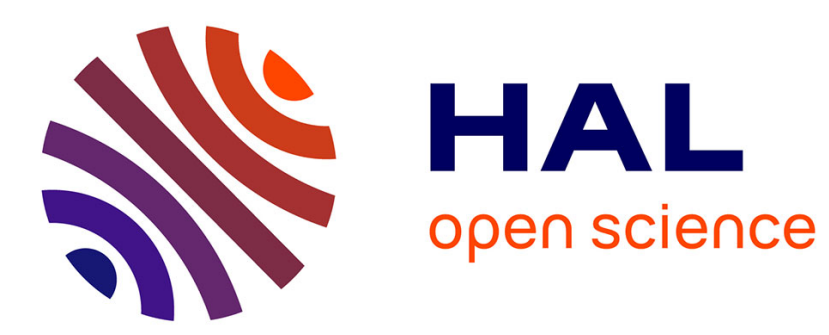

\title{
Laura Seguin, Apprentissages de la citoyenneté. Expériences démocratiques et environnement
}

\author{
Adrien Mollaret
}

\section{To cite this version:}

Adrien Mollaret. Laura Seguin, Apprentissages de la citoyenneté. Expériences démocratiques et environnement. Lectures, 2020, 10.4000/lectures.46142 . hal-03084304

\section{HAL Id: hal-03084304 \\ https://hal.science/hal-03084304}

Submitted on 21 Dec 2020

HAL is a multi-disciplinary open access archive for the deposit and dissemination of scientific research documents, whether they are published or not. The documents may come from teaching and research institutions in France or abroad, or from public or private research centers.
L'archive ouverte pluridisciplinaire HAL, est destinée au dépôt et à la diffusion de documents scientifiques de niveau recherche, publiés ou non, émanant des établissements d'enseignement et de recherche français ou étrangers, des laboratoires publics ou privés. 


\section{Laura Seguin, Apprentissages de la citoyenneté. Expériences démocratiques et environnement}

Adrien Mollaret

Doctorant en sociologie, Pacte, Université Grenoble Alpes.

$11 / 12 / 2020$

Cet ouvrage est la publication du travail de thèse de Laura Seguin ${ }^{1}$. Au croisement entre sociologie des mouvements sociaux, sociologie de l'action publique et sciences de l'éducation, il propose une réflexion sur «la fonction éducative de la participation » (p. 315). À travers une comparaison minutieuse de deux expériences de participation dans le domaine environnemental, l'ouvrage questionne à la fois l'amont de la participation, c'est-àdire le travail de définition, de cadrage et d'animation, et son aval, en interrogeant les effets de la participation sur les participants et les différentes parties prenantes. L’apport principal de l'ouvrage repose sur une double rupture avec les catégories traditionnelles dans la littérature scientifique, entre d'une part mouvements sociaux et «offre publique de participation $»^{2}$; et d'autre part entre citoyens « ordinaires » participants et les autres acteurs professionnels organisateurs ou animateurs de la participation (militants, associatifs, élus, acteurs publics).

Laura Seguin s'appuie sur une définition très ouverte de la participation comme «moments où les citoyens se réapproprient l'activité politique » au-delà de la participation électorale (p. 12). L'originalité de l'ouvrage réside dans la volonté d'inscrire sur un même continuum les expériences contestataires et les dispositifs institutionnels, de l'occupation d'une zone à défendre (ZAD) à l'engagement au sein d'un conseil citoyen (p. 9). Sans minimiser leurs différences, cette approche permet de mettre en lumière des processus et des tensions similaires à l'œuvre au sein de ces différents types d'expériences de participation politique.

\footnotetext{
${ }^{1}$ Soutenue en 2016 et lauréate du prix de thèse de la Commission nationale du débat public (CNDP) en partenariat avec le groupement d'intérêt scientifique (GIS) « Démocratie et participation ».

${ }^{2}$ Gourgues Guillaume, «Avant-propos : penser la participation publique comme une politique de l'offre, une hypothèse heuristique », Quaderni n ${ }^{\circ}$ 79, 2012, p. 5-12.
} 
Mobilisant un matériau ethnographique riche - qui mêle notes d'observation, portraits de participants et extraits d'entretiens - l'ouvrage propose une analyse croisée de deux expériences de participation. D'une part, une conférence citoyenne sur l'eau en Région Poitou-Charentes, mise en œuvre en 2011, comme exemple de dispositif institutionnel de participation publique commandité par les pouvoirs publics afin de trouver une issue à un conflit territorial latent autour de la gestion de la ressource en eau. D'autre part, une mobilisation contestataire spontanée et réactive, s'opposant au développement de l'exploitation du gaz de schiste en Ardèche, entre 2011 et 2013. Si la spécificité des enjeux environnementaux est abordée filigrane, en particulier le fait de «faire intervenir des savoirs scientifiques et techniques sources de controverses et comportant de nombreuses incertitudes » (p. 9), les questionnements ont une portée plus générale.

La première partie dresse le portrait de ces deux mobilisations, en revenant sur leur genèse et le travail de cadrage effectué par les «entrepreneurs » et les «artisans » de la participation. En entrant dans «les coulisses de la fabrique de la participation » (p. 18), Laura Seguin insiste sur les tensions qui traversent ces deux expériences et le nécessaire travail de négociation entre des représentations parfois contradictoires de la participation. Dans une seconde partie, Laura Seguin documente successivement les modalités d'apprentissage du conflit puis l'apprentissage de la construction d'accords collectifs - à travers l'apprentissage de la délibération collective, du langage des acteurs publics et des limites de la coopération. Elle démontre que les deux expériences «sont porteuses d'apprentissage du conflit comme de la délibération» (p. 315). Enfin, la dernière partie détaille les effets de la participation sur les participants, mais aussi sur les élus, les professionnels, les acteurs associatifs et les militants. Elle distingue trois « ressorts éducatifs » de ces expériences participatives: le socioconstructivisme, l'expérience et l'autonomie.

En comparant une forme de participation plus institutionnelle et une plus contestataire, l'autrice prend à bras le corps la question du conflit dans les expériences de participation ${ }^{3}$. L'enjeu théorique est stimulant: il s'agit de «repenser cette distinction entre dispositifs

\footnotetext{
${ }^{3}$ Bobbio Luigi et Melé Patrice, «Introduction. Les relations paradoxales entre conflit et participation », Participations, $\mathrm{n}^{\mathrm{o}}$ 13, 2015, p. 7-33.
} 
participatifs et mobilisations, entre délibération et conflit »(p. 11), en défendant la thèse d'une «imbrication des dimensions agonistiques et délibératives » (p. 18) dans toutes expériences de participation. L'approche ethnographique permet de dépasser l'opposition réductrice entre d'une part les dispositifs institutionnels de participation comme «tentative de réduction, voire d'épuisement du conflit»(p.11), et d'autre part les mobilisations contestataires comme «rapport et de force et visions du monde incompatibles » (p. 11). Contre cela, Laura Seguin propose une vision dynamique et processuelle selon laquelle toutes les expériences de participation, à des degrés et dans des temporalités différentes, sont traversées par des processus d'«ensauvagement » et de «domestication ${ }^{4}$. À l'encontre des idées reçues, l'ouvrage montre comment un dispositif institutionnel de participation comme une conférence citoyenne sur l'eau peut, dans certaines conditions, favoriser la mise en conflit et la montée en généralité en donnant à voir les oppositions. Parallèlement, il explique comment une mobilisation contestataire contre le gaz, traversée par le souci de produire une «parole entendable » et légitime, peut participer à un évitement du conflit dans une logique de coopération et de dialogue avec les pouvoirs publics.

Pour l'auteure, les trajectoires de ces deux expériences sont le résultat d'un rapport de force interne. $\mathrm{Au}$ sein de chaque expérience coexistent plusieurs conceptions de la participation et de ses objectifs. Dans le cas de la conférence citoyenne sur l'eau, ces oppositions sont matérialisées par les postures distinctes des deux concepteurs du dispositif, entre $1^{\prime} \mathrm{EPTB}^{5}$ de la Charente à la recherche d'un compromis et d'un consensus et l'Ifrée ${ }^{7}$ qui cherche à généraliser le débat et montrer les oppositions. De même, dans le cadre de la mobilisation ardéchoise, l'autrice documente les tensions entre un pôle plus tourné vers l'expertise et la discussion avec les pouvoirs publics et un pôle militant plus politisé. La trajectoire d'institutionnalisation et de professionnalisation de la mobilisation ardéchoise se traduira par une éviction volontaire et progressive de ces derniers.

4 Neveu Catherine, «Démocratie participative et mouvements sociaux : entre domestication et ensauvagement ? », Participations, ${ }^{\circ}{ }^{1}$, 2011, p. 186-209.

\footnotetext{
${ }^{5}$ Établissement public territorial de bassin.

${ }^{7}$ Institut de formation et de recherche en éducation à l'environnement.
} 
Ces deux expériences sont appréhendées avant tout comme des espaces de discussion rassemblant des «individus aux cultures politiques différentes » (p.209). En mobilisant une grille de lecture inspirée par les sciences de l'éducation, l'autrice documente les méthodes appliquées par les animateurs de la participation pour gérer cette cohabitation - comme le jeu de rôle, le débat mouvant ou le théâtre forum - qui participent à un travail d'« instruction du conflit » ${ }^{8}$ et d' «apprentissage de la délibération ». Elle en conclut que «ce n'est pas le conflit qui est exclu des arènes de discussion [...] mais ses modalités d'expression violentes conduisant à la rupture du dialogue » (p. 315).

Malgré certaines redondances - les mêmes constats ou résultats apparaissent parfois sous des formes très proches dans les trois parties - l'ouvrage tire un fil rouge original autour de «la dimension pédagogique et éducative d'une expérience démocratique » (p.13). L'importance de la restitution des matériaux de terrain participe à la richesse du récit, bien qu'ils prennent parfois le pas sur l'analyse et la prise de distance. L'approche par les apprentissages imposant un cadrage microsociologique, les pistes d'interrogation théoriques sur la place du conflit en démocratie, ainsi que sur le lien entre action publique et acteurs protestataires ${ }^{9}$, esquissées en introduction et dans la première partie, ne sont pas prolongées dans la suite de l'ouvrage.

${ }^{8}$ Morvan Alexia, Pour une éducation populaire politique : à partir d'une recherche-action en Bretagne, Thèse de doctorat, Paris 8, 2011.

${ }^{9}$ Dupuy Claire et Halpern Charlotte, «Les politiques publiques face à leurs protestataires », Revue francaise de science politique Vol. 59, $\mathrm{n}^{\circ}$ 4, 2009, p. 701-722. 